\title{
Numerical investigation of Rayleigh waves in layered composite piezoelectric structures using the SIGA-PML approach
}

\begin{abstract}
Existence of surface acoustic waves(SAW) on a piezoelectric layer with the half-infinite elastic layers structures is investigated. This structures belong to open waveguide with unbounded boundary in the transverse directions. Except for trapped modes, leaky modes have often been considered in SAW applications, which require waves of low attenuation in order to maximize the propagation distance. Therefore, we develop an another formulation of piezoelectric layer structures for the computation of trapped and leaky modes in open waveguides. This method combines the so-called semi-analytical isogeometric analysis and a perfectly matched layer technique (SIGA-PML). The comparison between semi-analytical finite element (SAFE-PML) and SIGA-PML is given, in order to show the effective and accuracy of SIGA-PML. Finally, we analyze propagation properties of Rayleigh waves and discuss the impact of the thickness of $\mathrm{Cu}$ films on the dispersive relationships.
\end{abstract}

\section{Introduction}

In 1965, White and Voltmer developed the first device[1], which generated surface acoustic wave (SAW) involving configuration of a thin piezoelectric layer over a substrate. Since then, a considerable amount of interest has focused on harnessing SAW technology. SAW have been employed in surface science and seismology, acoustic filter, and nondestructive evaluation, and so on [2, 3]. Up to now, many researchers and engineers have being meanly to focus on SAW techniques of cell biology and medicine. And, it was found that SAW has a effectively functionality with separating cell[4]. Among different structures, a piezoelectric layer structure in SAW sensors was considered to be more competent for it generated electrical signals of Rayleigh and Love waves.

Generally, the elastic noduli of piezoelectric materials can be hundreds or even thousands times larger than those of substrates, which means mean that the bulk wave velocity of films exceeds that of the substrate. Hence, these systems generate some leaks leaky mode, except for trapped and radiation modes[5-7]. Leaky modes can be described as imperfectly waves. Similar to trapped mode, leaky modes have the potential to well represent the continuum over the surface of the waveguide. Their unusual behavior is that their amplitudes exponentially growing in the transverse direction, while decaying along the axis. Their some energies will be transferred into the substrate. Such attenuation can strongly limit the application of SAW techniques. An important issue to resolve for the development of efficient SAW devices, therefore, is how to achieve leaky modes of low attenuation to sustain the strength of exciting signal.

The investigation of surface wave of multilayered piezoelectric systems becomes essential to be able to guide the design and fabrication in favor of obtaining a better performance or new functionalities of SAW sensors. In 1968, Bleustein[8] has employed an analytical model to consider dispersive properties of surface wave of a piezoelectric half with various electrical boundary conditions. The rotation effect on surface wave propagation in a ceramics PZT-5H half space has been discussed by Qing Jiang et al[9].

\footnotetext{
* Corresponding author

Email address: liangyingjing@126.com (Yingjing Liang)
} 
Chai and $\mathrm{Wu}[10]$ have shown SAW of pre-stressed piezoelectric structures using the LotheCBarnetts integral formalism and analyzed the pre-stressed effect on dispersive relationship. Du et al.[11] have developed the analytical model of Love wave in a functionally graded piezoelectric material layer with a substrate. Pang. et al[12] has investigated propagation properties of Rayleigh-type surface waves in a piezoelectric-piezomagnetic layered half-space. Cao. et al[13] studied dispersive properties of Rayleigh surface waves in a transversely isotropic graded piezoelectric half-space by using the WentzelCKramersCBrillouin (WKB) technique. And, they[14] presented an analytical model of Rayleigh surface waves in a piezoelectric wafer with subsurface damage and discussed their dispersive relationship. Legendre and Laguerre polynomial approaches were employed to study wave propagation in layered magnetoelectro-elastic media by Mater et al[15]. Zhang[16] discussed the surface effect on the piezoelectric nanofilm with a elastic substrate. Enzevaee and Shodja[17] studied analytically Love and Rayleigh surface wave propagations in a semi-infinite medium with face-centered cubic single crystal structure, based on surface elasticity theory. In all these previous works, methods based on the matrix formalism, analytical models or have been used, yet limited to simple geometries and lack of expendability and portability. This paper proposed the approach combing the so-call semi-analytical isogeometric analysis (SIGA) and a perfectly matched layer (PML) technique, to study wave propagation of Rayleigh surface waves in a PVDF layer with a half-space elastic substrate.

SIGA was employed to computer the dispersive curves of close waveguides[18, 19]. This method combines the best aspects of semi-analytical approach and IGA. In this work the isogeometric analysis (IGA) [20-22] is employed to describe and model the geometry of structure by NURBS basis functions for the high smoothness and the flexible parameterizations. The semi-analytical theory, is exploited to study wave propagation with translational invariance[23]. Assuming harmonic functions of axis and time, this method can reduce the two-dimensional problem to the one-dimensional one, so that only the transverse section is needed need to be meshed, to vastly reduce the computational cost.

The above SAW structures with an elastic substrate can be regarded as open waveguides. Yet for unbounded boundary, a difficulty a problem occurs for the region of the transverse direction is infinite, while traditional numerical methods are employed. Lowe et al.[24-26] proposed the absorbing layer (AL) method, where a viscoelastic layer is added to the truncated boundary. In order to simulate the attenuation of energy along the depth, the damping coefficient of the viscoelastic layer also increases with the increase of thickness. As longer absorbing layers are needed to reduce artificial reflections, the degrees of freedom are extensively increased. Mazzotti et al. have developed the regularized 2.5D boundary element method to analyze dispersive relationship of three-dimensional elastic waveguides embedded in a solid[27] or in a fluid[28]. This method requires to obtain the fundamental solution for BEM and yields nonlinear eigenproblems, which take higher computation cost. More recently, PML is coupled with SAFEM to model open plate[29] and three-dimensional solid[30, 31] waveguides, which can strongly aattenuate elastic wave without refelction. Compared to AL, the PML method allows to reduce the artificial length and obtain more accuracy results for waveguide problems. Its advantage is that the computational continuum domains for governing equations are mapped into the complex spatial space.

This paper focuses on numerical model of surface waves in a PVDF layer with a half-space elastic substrate using the SIGA-PML approach. The comparison between SIGA-PML and SAFE-PML is studied for a elastic open waveguide. The propagation properties of Rayleigh waves are analyzed. Finally, we discussed the impact of the $\mathrm{Cu}$ thickness and elastic modulus on the dispersive relationships of SAW.

\section{Formulation of SIAG-PML}

Consider a PVDF layer with a half-space elastic substrate, as depicted Fig.1. The top and bottom of PVDF are deposed by a $\mathrm{Cu}$ film as the electrical machine, respectively. The such structure is regarded as a open waveguide. It is assumed that the general displacements(elastic displacement, electric potential) and stresses of the interfaces between layers are continuous and the elastic stresses are free on the top and bottom surfaces. Waves propagates along the $x$-axis direction. This section will give the formulation of SIGA-PML for the such structure. 

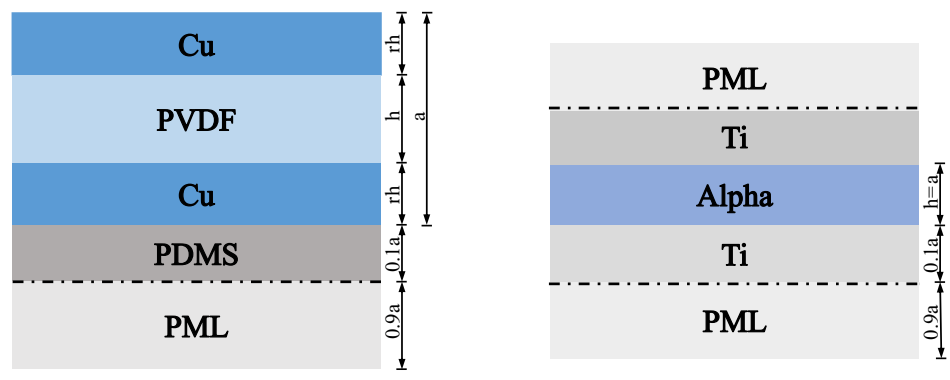

Fig. 1. Sketch map of a Table.1Cu-PVDF-Cu waveuide

\subsection{NURBS-based isogeometric element}

In analogy to the isoparametric element, the basis-field function of IGA is identical with the shape function of the geometry model. Following the paradigm of IGA, the physical and geometrical fields both are discretized with using the NURBS basis functions. This work focuses on eigenmodes of waveguide problems, so that external forces are then discarded. Therefore, the cross section of a twodismensional waveguide only need to be described by one-dimensional NURBS curve elements after using the semi-analytical method.

The cross section of a one-dimensional structure is shown by $n$ curves of degree $p$, where each one curve is corresponding to a path. For the parametric of a given patch, the knot vector is a set of non-decreasing values, given as

$$
\boldsymbol{\Xi}=\left[\xi_{1}, \xi_{2}, \cdots, \xi_{n_{\xi}+p+1}\right]
$$

The entries of $\boldsymbol{\Xi}$ describe the integral regions of the elements, i.e. each knot span $\left[\xi_{i}, \xi_{i+1}\right]$ for $i \in$ $\left[p, \cdots, n_{\xi}\right]$, indexed by a element $e$, has $p+1$ non-zero B-spline basis functions. The B-spline basis function $N_{i, p}(\xi)$ with $p=1$ is described as

$$
N_{i, p}(\xi)= \begin{cases}1, & \text { if } \xi_{i} \leqslant \xi<\xi_{i=1} \\ 0, & \text { else }\end{cases}
$$

And they with $p>1$ are defined recursively by the Cox-de Boor formula

$$
N_{i, p}(\xi)=\frac{\xi-\xi_{i}}{\xi_{i+p}-\xi_{i}} N_{i, p-1}(\xi)+\frac{\xi_{i+p+1}-\xi}{\xi_{i+p+1}-\xi_{i+1}} N_{i+1, p-1}(\xi) \text { Here },
$$

Here, the open uniform knot vectors only are considered, and the B-spline basis functions are $C^{p-1}$ continuous in the whole patch region. Fig.2 illustrates one-dimensional quadratic B-spline basis functions. NURBS can be built from the B-spline basis functions. Univariate NURBS basis functions are expressed as

$$
R_{i}(\xi)=\frac{w_{i} N_{i}(\xi)}{\sum w_{j} N_{j}(\xi)}
$$

under consideration of the additional value weight $w_{i}$ with respect to $N_{i}$. There is a one-to-one correspondence between the control point index $\mathbf{P}_{i}\left(x_{i}, y_{i}, z_{i}\right)$ and the NURBS function $R_{i}$ on path level. The curve on cross section is interpolated by

$$
\mathbf{C}(\xi)=\sum_{i=1}^{n_{\xi}} R_{i}(\xi) \mathbf{P}_{i}
$$

Furthermore, they also are applied into the approximation of the physical field. 


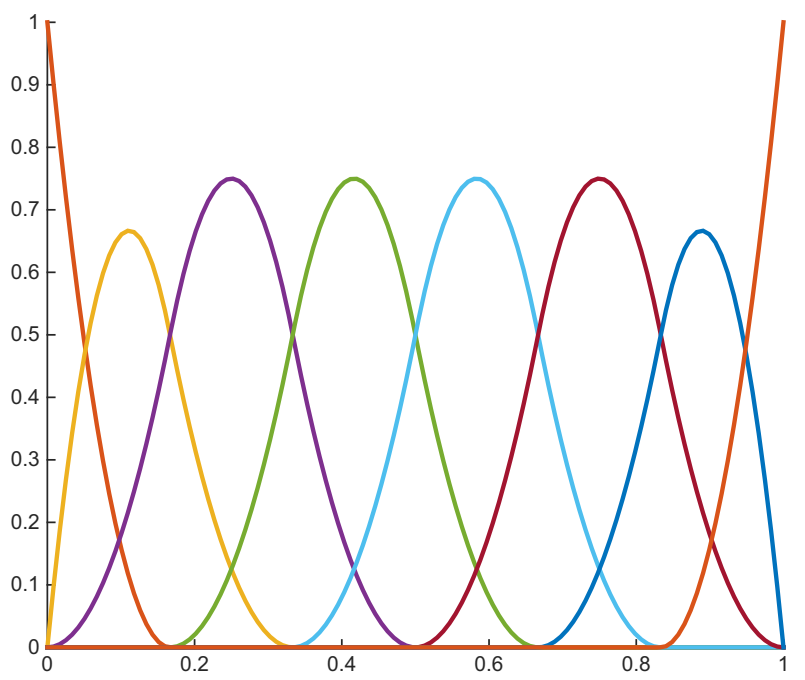

Fig. 2. Set of B-spline basis functions for $\Xi=\left[\begin{array}{lllllllllll}0 & 0 & 0 & \frac{1}{6} & \frac{1}{3} & \frac{1}{2} & \frac{2}{3} & \frac{5}{6} & 1 & 1 & 1\end{array}\right]$

\subsection{Basic equations}

For one waveguide, only extra small strains need to be considered. Assuming that material's parameters polar along the $\mathrm{y}$-axis direction. General piezoelectric material's constitutive equation yields

$$
\begin{aligned}
& \sigma_{i j}=C_{i j k l} \varepsilon_{k l}-e_{k i j} E_{k} \\
& D_{i}=e_{i k l} \varepsilon_{k l}+v_{i k} E_{k}
\end{aligned}
$$

where $\sigma_{i j}$ and $D_{i}$ are the stress tensor and electric displacement, respectively; $\epsilon_{k l}$ and $E_{k}$ are the strain tensor and electric field intensity, respectively; $C_{i j k l}, e_{i j k}$ and $v_{i k}$ represent the elastic constant, piezoelectric constant and dielectric constant, respectively; Einstein summation convention are used, where $i, j, k$ and $l=1,2,3$, corresponding to $r, \theta, z$, respectively

We define the generalized stress vector $\overline{\boldsymbol{\sigma}}$ and strain vector $\overline{\boldsymbol{\varepsilon}}$. The generalized constitutive equations can be rewritten as

$$
\bar{\sigma}=\mathbf{H}^{*} \bar{\varepsilon}
$$

with the generalized constitutive matrices

$$
\mathbf{H}^{*}=\left[\begin{array}{cc}
\mathbf{C} & -\mathbf{e}^{T} \\
\mathbf{e} & \boldsymbol{v}
\end{array}\right] \quad \mathbf{H}=\left[\begin{array}{cc}
\mathbf{C} & -\mathbf{e}^{T} \\
-\mathbf{e} & -\boldsymbol{v}
\end{array}\right]
$$

The relationship between the generalized strain and generalized displacement can be expressed as

$$
\bar{\varepsilon}=\mathbf{L}_{x} \overline{\mathbf{u}}_{, x}+\mathbf{L}_{0} \overline{\mathbf{u}}
$$

where the generalized displacement $\overline{\boldsymbol{u}}=\left[\begin{array}{lllll}u_{1} & u_{2} & u_{3} & -\phi\end{array}\right]^{T}$, consisting of the displacements $u_{i}$ and the electric potential $\phi$; The differential operator $\mathbf{L}_{x}, \mathbf{L}_{0}$ can be given in Appendix, respectively.

The linear theory of piezoelectricity is derived from assumption that the infinitesimal strain tensor is the symmetric portion of the spatial gradient of the mechanical displacement and electric fields are small. The charge equations of electrostatics are coupled to the mechanical deformations by using a modified Lagrangian density given by

$$
\mathcal{L}=\frac{1}{2} \rho \dot{\mathbf{u}}^{2}+\mathcal{H}(\mathbf{E}, \boldsymbol{\varepsilon})
$$

where the subscript ' denotes the material time derivative; $\mathcal{H}$ is the electric enthalpy density function, 
which depend on the components of the Cauchy-Lagrangian strain tensor and the electric field; The electric enthalpy density can be further given as

$$
\mathcal{H}(\mathbf{E}, \mathbf{T})=0.5 \varepsilon: \mathbf{C}: \varepsilon-\varepsilon: \mathbf{e E}-0.5 \mathbf{E} \boldsymbol{v} \mathbf{E}
$$

Based on Hamilton s principle, a set of governing equations can be developed for piezoelectric composite structures. Employing the above definitions, and neglecting body forces, the variation of the action functional based on the Lagrangian density can be formulated as

$$
\int_{V} \boldsymbol{\delta} \varepsilon^{T} \mathbf{H} \boldsymbol{\sigma} d V+\int_{V} \boldsymbol{\rho} \delta \mathbf{u}^{T} \ddot{\mathbf{u}} d V=0
$$

The above equation in piezoelectric elastic system presents variations of the stored electric enthalpy and also is applied to describe the elastic wave problem of the complex domain.

\subsection{Dispersive equation of SIGA-PML}

Here, Perfectly Matched Layer (PML) is set around the composite structures with a certain thick as an absorption layer. It can avoid the reflection of any wave signals. Compared to infinite element method, low-reflecting boundary condition and absorbing layers by increasing damping, PML is not sensitive to incident angle and frequency of waves, and it is good at the manipulation of high-frequency waves without numerical reflections. Until now, PML has been widely used in electromagnetism, Geophysics, Ultrasound and other fields. In this work, the energy of elastic waves in the infinite plates may leak into the substrates. For the sake of computing wave characteristics, the truncated boundary condition must be introduced by the PML. Hence, the PML maps the original geometry into a new complex coordinate system with a stretching function

$$
y(y)=\int_{0}^{y} \gamma(\xi) d \xi
$$

where $\gamma(\xi)$ represents a non-zero, continuous and complex stretching function, that is the PML profile and it follows $\gamma(y)=1$ for $y \leq h_{1}, \operatorname{Im}(y)>0$ for $y>h_{1}$.

The variation of arbitrary function $\sim f$ can be written as

$$
\tilde{f}(y)=f(y), d \tilde{y}=\gamma(d) d y, \frac{\partial \tilde{f}}{\partial \tilde{y}}=\frac{1}{\gamma(d)} \frac{\partial f}{\partial y}
$$

Via using the above expression, $\mathbf{L}_{s}$ can be obtained by

$$
\mathbf{L}_{s}=\frac{1}{\gamma} \mathbf{L}_{y} \frac{\partial}{\partial y}
$$

Using the above NURBS basis functions, the structure along the y-axis direction is parameterized and the mapping from the local parametric to the global coordinates

$$
y=\mathbf{R}(\eta) \mathbf{P}(x)
$$

in which, $\mathbf{R}(\eta)$ means the NURBS basis function, $\mathbf{P}(x)$ is the control points, $y$ is the actual Cartesian coordinates and $\eta$ is the parametric coordinates. The Jacobian matrix $\mathbf{J}$ between the physical and parametric coordinates is

$$
\left[\frac{\partial \mathbf{R}}{\partial \eta}\right]=J\left[\frac{\partial \mathbf{R}}{\partial y}\right], \mathbf{J}=\left[\frac{\partial y}{\partial \eta}\right]
$$

In terms of the isoparametric thought, the displacement at a point can be expressed as

$$
\overline{\mathbf{u}}(x, y, t)=\mathbf{R}(\eta) \overline{\mathbf{U}}(x, t)
$$


where $\overline{\mathbf{U}}(\mathbf{x}, \mathbf{t})$ denotes the generally displacement vectors of control points. According Eq.15 and Eq.16, the generalized strain is given as

$$
\bar{\varepsilon}=\mathbf{B}_{1} \overline{\mathbf{U}}(x, t)+\mathbf{B}_{2} \overline{\mathbf{U}}_{, x}(x, t)
$$

with the generally strain matrices

$$
\mathbf{B}_{1}=\frac{1}{|\mathbf{J}|} \mathbf{L}_{s} \mathbf{R}_{, \eta} \quad \mathbf{B}_{2}=\mathbf{L}_{x} \mathbf{R}(\eta)
$$

We can assume its displacement is independent of the time harmonic $e^{-j \omega t}$, where $t$ is the time and $\omega$ denotes the angular frequency. After the axial Fourier transformation, the generally displacement can be described as

$$
\overline{\mathbf{U}}(x, t)=\hat{\mathbf{U}} e^{j(k x-\omega t)}, \delta \overline{\mathbf{U}}(x, t)=\delta \hat{\mathbf{U}} e^{j(-k x+\omega t)}
$$

where $k$ is the wavenumber along the $x$ direction. $j$ represent imaginary unit

After combing with Eq.16 and Eq.19, substituting Eq.21 into Eq.12, the dispersion equation yields

$$
\mathbf{E}_{1} \hat{\mathbf{U}}+j k\left(\mathbf{E}_{2}-\mathbf{E}_{1}^{T}\right) \hat{\mathbf{U}}+k^{2} \mathbf{E}_{3} \hat{\mathbf{U}}-\omega^{2} \mathbf{M}_{\mathbf{0}} \hat{\mathbf{U}}=0
$$

where $\hat{\mathbf{U}}$ is the amplify of the generally displacement of control points. The subscript $T$ denotes the transpose. The stiffness matrices and mass matrix are given as

$$
\begin{array}{ll}
\mathbf{E}_{1}=\int_{\zeta} \mathbf{B}_{1}^{T} \overline{\mathbf{H}} \mathbf{B}_{1} \gamma|\mathbf{J}| d \eta & \mathbf{E}_{2}=\int_{\zeta} \mathbf{B}_{1}^{T} \overline{\mathbf{H}} \mathbf{B}_{2} \gamma|\mathbf{J}| d \eta \\
\mathbf{E}_{3}=\int_{\zeta} \mathbf{B}_{2}^{T} \overline{\mathbf{H}} \mathbf{B}_{2} \gamma|\mathbf{J}| d \eta & \mathbf{M}_{0}=\int_{\zeta} \rho \mathbf{R}^{T} \mathbf{R} \gamma|\mathbf{J}| d \eta
\end{array}
$$

with the generalized density matrix

$$
\boldsymbol{\rho}=\left[\begin{array}{lll}
\rho & 0 & 0 \\
0 & \rho & 0 \\
0 & 0 & 0
\end{array}\right]
$$

Note that the dispersion equation Eq.22 becomes a quadratic eigenvalue problem related to wavenumber $k$, for a set of given frequencies. Via the matrix transformation, Eq.22 can be simplified into a linear eigen-system

$$
-\mathbf{Z} \boldsymbol{\psi}=\lambda \boldsymbol{\psi}, \lambda=i k
$$

with

$$
\mathbf{Z}=\left[\begin{array}{cc}
\mathbf{E}_{3}^{-1} \mathbf{E}_{2}^{T} & -\mathbf{E}_{3}^{-1} \\
\omega^{2} \mathbf{M}_{\mathbf{0}}-\mathbf{E}_{1}+\mathbf{E}_{2} \mathbf{E}_{3}^{-1} \mathbf{E}_{2}^{T} & -\mathbf{E}_{2} \mathbf{E}_{3}^{-1}
\end{array}\right], \boldsymbol{\psi}=\left(\begin{array}{c}
\hat{\mathbf{U}} \\
\hat{\mathbf{Q}}_{n}
\end{array}\right)
$$

where $\hat{\mathbf{Q}_{n}}$ represents the distributed force on the cross-sections of waveguides and is rewritten as

$$
\hat{\mathbf{Q}}_{n}=\lambda \mathbf{E}_{3} \hat{\mathbf{U}}+\mathbf{E}_{2}^{T} \hat{\mathbf{U}}
$$

Owing to the symmetry matrices $\mathbf{E}_{1}, \mathbf{E}_{2}, \mathbf{M}_{0}$ and the Hamiltonian matrix $\mathbf{Z}$, there exist $n$ pairs of eigenvalues, like this $\left(k_{i}, \hat{\mathbf{U}}_{\mathbf{i}}\right)$ and $\left(-k_{i}, \hat{\mathbf{U}}_{\mathbf{i}}\right)(i=1,2, \ldots, n)$, which represent positive- and negativepropagating waves, respectively.

The phase velocity $c_{p}$ is given by $c_{p}=\omega / k$, and the group velocity is defined by

$$
c_{g}=\frac{d \omega}{d k}=j \frac{d \omega}{d \lambda}
$$

For a certain eigenvalue $\lambda$, the left- and right- eigenvectors are $\hat{\mathbf{U}}_{\mathbf{L}}$ and $\hat{\mathbf{U}}_{\mathbf{R}}$. The differential form of Eq.22 can be given by

$$
\left(\left(\mathbf{E}_{2}-\mathbf{E}_{2}^{T}\right)+2 \lambda \mathbf{E}_{3}-2 j \omega \mathbf{c}_{\mathbf{g}} \mathbf{M}\right) \hat{\mathbf{U}}_{R}=0
$$


Multiplying by $\hat{\mathbf{U}}_{\mathbf{L}}^{H}$, the above equation is

$$
\hat{\mathbf{U}}_{L}^{H}\left(\left(\mathbf{E}_{2}-\mathbf{E}_{2}^{T}\right)+2 \lambda \mathbf{E}_{3}-2 j \omega \mathbf{c}_{\mathbf{g}} \mathbf{M}\right) \hat{\mathbf{U}}_{R}=0
$$

where the superscript $H$ denotes the conjugate transpose. The the formulation of group velocity can be written by

$$
\mathbf{c}_{\mathbf{g}}=j \frac{\hat{\mathbf{U}}_{L}^{H} \hat{\mathbf{Q}}_{R}-\hat{\mathbf{Q}}_{L}^{T} \hat{\mathbf{U}}_{R}}{2 \omega \hat{\mathbf{U}}_{L}^{T} \mathbf{M} \hat{\mathbf{U}}_{R}}
$$

and

$$
\hat{\mathbf{Q}}_{R}=\left(\lambda \mathbf{E}_{3}+\mathbf{E}_{2}^{T}\right) \hat{\mathbf{U}}_{R}, \quad \hat{\mathbf{Q}}_{L}=\left(-\lambda \mathbf{E}_{3}+\mathbf{E}_{2}^{T}\right) \hat{\mathbf{U}}_{L}
$$

\section{Stretching function and Mode filtering}

The PML technique has three parameters: the boundary $h_{l}$, the PML thickness $h_{p m l}$ and the absorbing function $\gamma$, as shown in Fig.3. It is very critical to maximize attenuation of travel waves and reduce reflections by optimizing these parameters. expression yields

$$
\hat{\gamma}=\frac{1}{d} \int_{d}^{d+h 1} \gamma(\xi) d \xi
$$

from Eq.??, when $\arg k_{l}+\arg k_{s}>0(\arg \in[-\pi, \pi])$, the leaky modes decay exponentially through the PML. As the arg $\hat{\gamma}$ increases, the complex domain will be expanded and the attenuation also becomes more and more serious with $\left|k_{l}\right||\hat{\gamma}|$ increasing. To a certain extent, the stretching function should be chosen properly to ensure good results. We choose the function as follows

$$
\gamma(y)= \begin{cases}1, & \text { when } \xi \leq h_{l} \\ 1+3(\hat{\gamma}-1)\left(\frac{y-h_{l}}{h_{p m l}}\right)^{2}, & \text { when } \xi>h_{l}\end{cases}
$$

Note that the above stretching function is independent of frequencies in order to avoid the calculation of stiffness matrices at each $\omega$.

Generally, open waveguides can generate trapped modes, leaky modes and radiation modes. Trapped modes travel without attenuation, whose energies only focus on the core region of waveguide and do not leak into the surrounding media. By contrast, radiation modes can be either oscillation and evanescent in the transverse directions, sometimes regarded as resonator modes, so that their only have extremely short propagation distances. In practice, radiation modes are of interest to application. Thereby, the technique of mode filtering is required to remove the radiation modes in post-processing. Based on the ratio of energy flux in the waveguide region, the criterion of mode filtering is provided as

$$
\frac{\left|\mathbf{T}_{k p m l}\right|}{\left|\overline{\mathbf{T}}_{k}\right|}<\beta_{\max }
$$

where $\beta_{\max }$ is a user-defined parameter, $\bar{T}$ is the time average kinetic energy, given as

$$
\mathbf{T}=\frac{\omega^{2}}{4} \int_{s} \rho \overline{\mathbf{u}}^{T} \cdot \overline{\mathbf{u}} \gamma d s
$$

The total kinetic energy $\mathbf{T}_{k}$ can be computed as

$$
\mathbf{T}_{k}=\frac{\omega^{2}}{4} \hat{\mathbf{U}}^{H} \mathbf{M}_{\mathbf{0}} \hat{\mathbf{U}}
$$

This expression's integration domain is corresponding to the whole transverse region. The PML kinetic energy $\mathbf{T}_{k p m l}$ can be solved to integrate in the $h_{p m l}$ domain. 


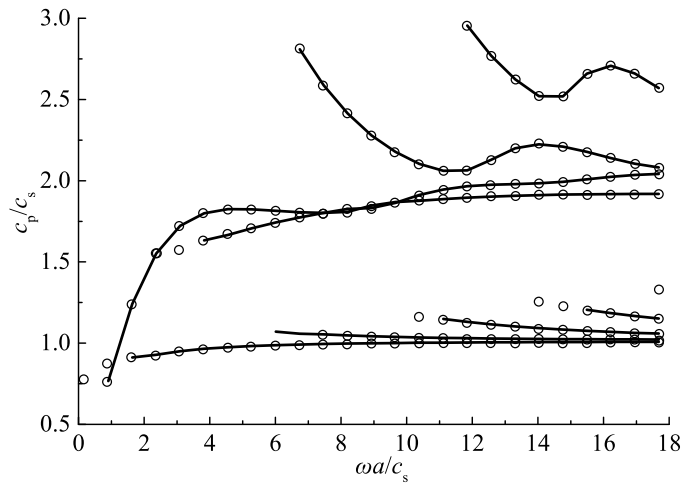

(a) Phase velocity curves

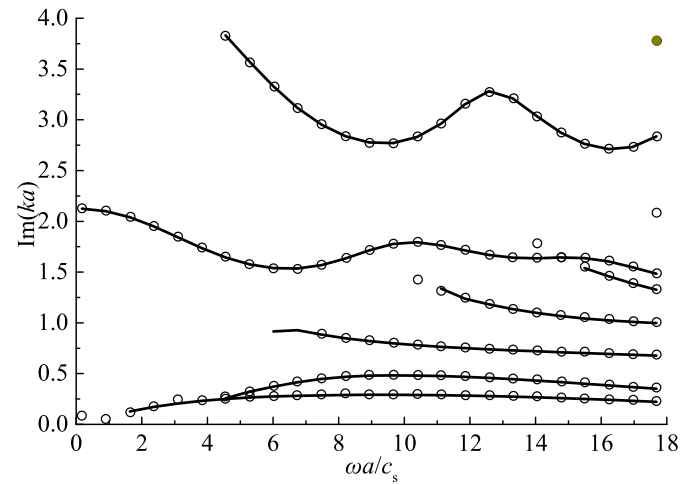

(b) Imaginary part curves of wavenumber

Fig. 3. Comparison between SAFE-PML (circles) and SIGA-PML (continuous lines) for elastic open waveguide

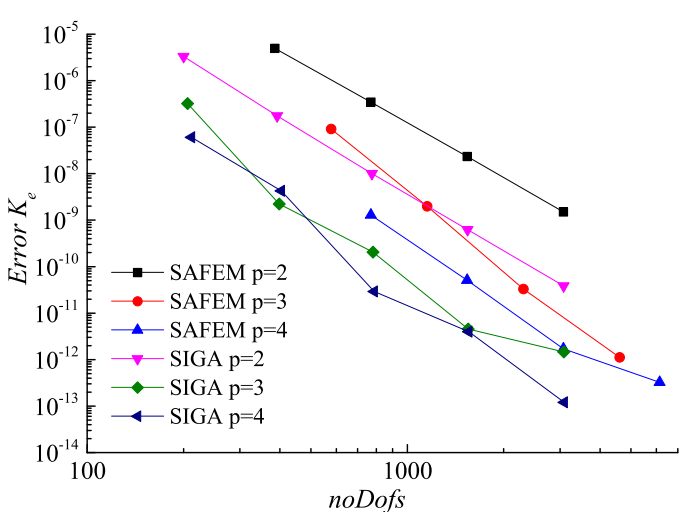

(a) The first mode

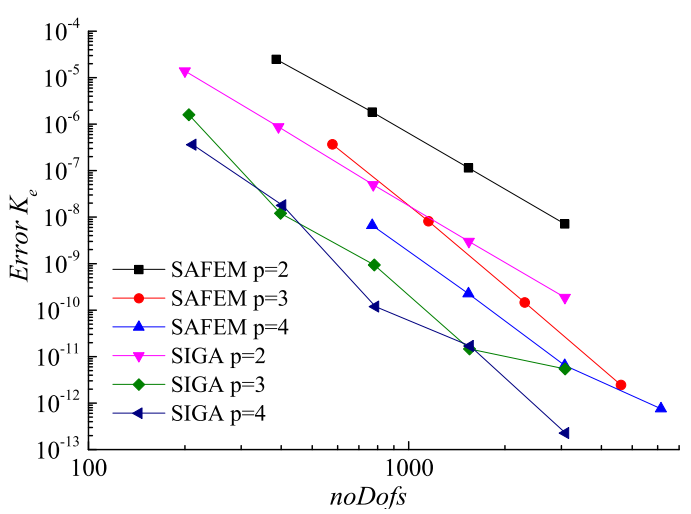

(b) The second mode

Fig. 4. Numerical convergence curves of SAFE-PML and SIGA-PML

\section{Results and Discussions}

Firstly, this section employs the above formulation to calculate a case, in order to provide the effective and validation of SIGA-PML, as shown in Fig.3. Then, this method is applied to analysis Rayleigh waves in the piezoelectric layer with an elastic substrate. The piezoelectric layer is used as the PVDF materialwhich is more flexible and stretched to adapt larger deformation compared with the PZT material.

\subsection{Elastic open waveguide}

The method proposed in this paper also was employed to analyze the dispersive relationship of elastic open waveguides. Its dispersive equation can be obtained via removing the row and column whose all elements equal to zeros, after piezoelectric and dielectric parameters of materials given as zeroes. Without loss of generality, we choose variables adimensionalised with some parameters, length $h$ and time $h / c_{s}$. The material properties of the Ti-Alpha-Ti waveguide are shown in Table.1. Thus, the dimensionless frequency and wavenumber are given by $\Omega=h \omega / c_{s}$ and $K=k h$ respectively, where $h$ and $c_{s}$ are the thickness of core layered waveguide and the shear wave velocity $\sqrt{C_{44} / \rho}$, respectively.

Fig.1 gives the geometry and material distribution of elastic open waveguide. It is taken from the work of Treyssde. Clearly, results of phase velocity and attenuation curves obtained by using SIGA-PML for Rayleigh waves are identical with these of SAFE-PML, shown in Fig.3, where these two methods adopt the same PML parameters, where $\gamma=1+4 j$ and $\beta_{\max }=0.9$. In SIGA-PML, NURBS with $p=2$ are utilized as basis functions to discrete the transverse section. 
Table 1

Material properties on $\mathrm{Cu}, \mathrm{PVDF}$ and PDMS

\begin{tabular}{c|cccccc}
\hline Properies & E & Poison ratio & $v_{11}$ & $e_{13}$ & $e_{33}$ & $\rho$ \\
\hline PVDF & 2 & 0.326 & 23 & 23 & 23 & 1780 \\
Cu & 119 & 0.29 & - & - & - & 8900 \\
PDMS & $10^{-5}-10^{3}$ & $0.40-0.5$ & - & - & - & 953 \\
Ti & 2.716 & 0.3 & - & - & - & 4460 \\
Alpha & 3.228 & 0.3 & - & - & - & 4460 \\
\hline
\end{tabular}

Units $E\left(10^{9} \mathrm{~N} / \mathrm{m}^{2}\right), v_{i j}\left(10^{-10} \mathrm{~F} / \mathrm{m}^{2}\right), e_{i j}(\mathrm{C} / \mathrm{m}), \rho\left(\mathrm{kg} / \mathrm{m}^{3}\right)$

To evaluate the accuracy of SIGA-PML, results at $\Omega=1$ for the Ti-Alpha-Ti waveguide has been computed with a sequence of refined meshes. The expression of posterior error estimation related to wavenumbers are given as

$$
K_{e}=\frac{K_{i}-K_{\bar{i}}}{K_{\bar{i}}}
$$

where $K_{\bar{i}}$ denotes the dismensionless wavenumber of the refined mesh. Fig.4 gives convergence curves of SIGA-PML and SAFE-PML methods for the first-order and second-order modes, where the quadratic, cubic and quartic NURBS and Lagrangian elements are employed. We can find out that these calculations are in good agreement with the difference less than $10^{-2}$ percent. And these curves are approximate beelines on the dual logarithmic coordinate diagram. Obviously, SIGA-PML has a faster convergent rate than SIGA-PML. For the same order basis functions, NURBS elements show the higher smoothness and continuity, compared with Lagrangian elements. When the order of the basis functions is elevated, more accurate solutions are obtained. At the same noDofs, solutions of the first-order mode are more accuracy than those of the second-order mode.

\subsection{PVDF with the PDMS substrate}

In this part, SIGA-PML is applied to study propagation properties of of Rayleigh waves for CuPVDF-Cu waveguide. Table.1 gives material parameters of $\mathrm{Cu}, \mathrm{PVDF}$ and PDMS. As variables adimensionalised, the length $h$ equals to $1 \mathrm{~mm}$ and the velocity $c_{s}$ is computed according to metrical parameters of $\mathrm{Cu}$.

Fig.5 gives spectrum, phase and group velocity curves of one $\mathrm{Cu}-\mathrm{PVDF}-\mathrm{Cu}$ waveguide, where the thickness of $\mathrm{Cu}, \mathrm{PDVF}, \mathrm{PDMS}$ and PML are $0.1 \mathrm{~mm}, 1 \mathrm{~mm}, 0.1 \mathrm{~mm}, 0.12 \mathrm{~mm}$ and $1.08 \mathrm{~mm}$, respectively, and $E$ of PDMS is $1.5 \mathrm{MPa}$. Clearly, compared with results of a piezoelectric film deposed on a elastic substrate, the dispersive behavior becomes more complex. Here, we mainly analyze dispersive properties of the first-five modes, named via the sequence method, shown in Fig.5. The first-two modes have strong in the low-frequency region, while their curves trend to straight line forms in the highfrequency region. In other words, the dispersive properties of the first-two mode are not obvious in the high-frequency region. When this structure are used as SAW devices, exciting signals of Rayleigh wave are relatively stable in the above frequency range.

In Fig.6(a), we can observe that spectrum curves of $1^{\text {st }}$ and $2^{\text {nd }}$ modes approach each other closely and suddenly veer away again, each one tracking down the locus of the other. All of the characters of two modes are exchanged, including phase velocities, group velocities, and wavestructures. The process keeps always smooth and albeit abrupt. From Fig.5(b), a veering curve of frequency spectrum always results in a jumping area in the group velocity curve corresponding to Fig.6(a), actually identified as the notch frequency. Thus, modal transition between $1^{\text {st }}$ and $2^{\text {nd }}$ modes occurs around $\Omega=1$. Thus , the jumping level between $2^{\text {st }}$ and $4^{\text {nd }}$ modes becomes more obvious, shown in Fig.5(c).

Distribution of the mechanical displacement and electric potential of first-five modes at $\Omega=2$ are given in Fig.7. We discover that $u_{1}$ and $u_{3}$ displacement in the PML layer almost equal to zeros, further to present the validation of SIGA-PML. The change trend of first-two modal displacement becomes more transparent than that of last three modes. And, the intensity of displacement distribution increases as the modal order increases. Compared to mechanical displacement, the distribution of electrical potential shows more simple, which are approximated by the trigonometric functions. 


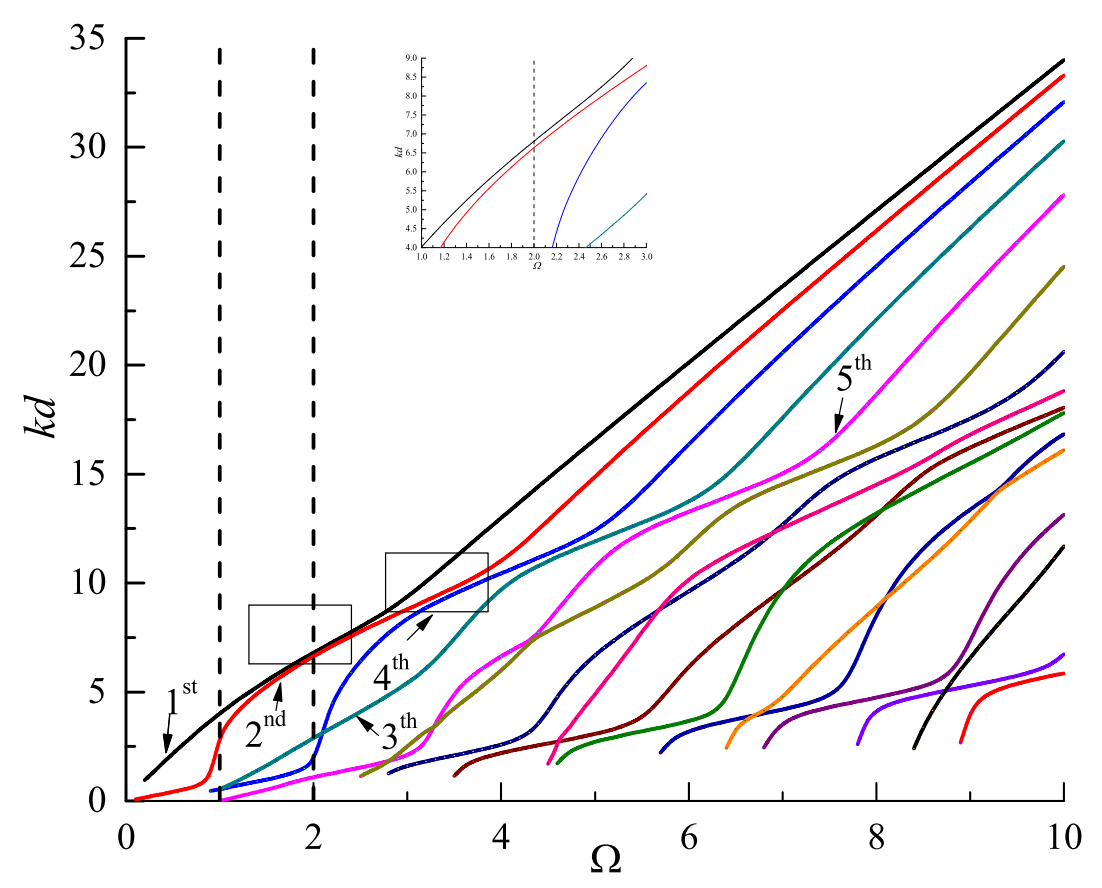

(a) Spectrum curves

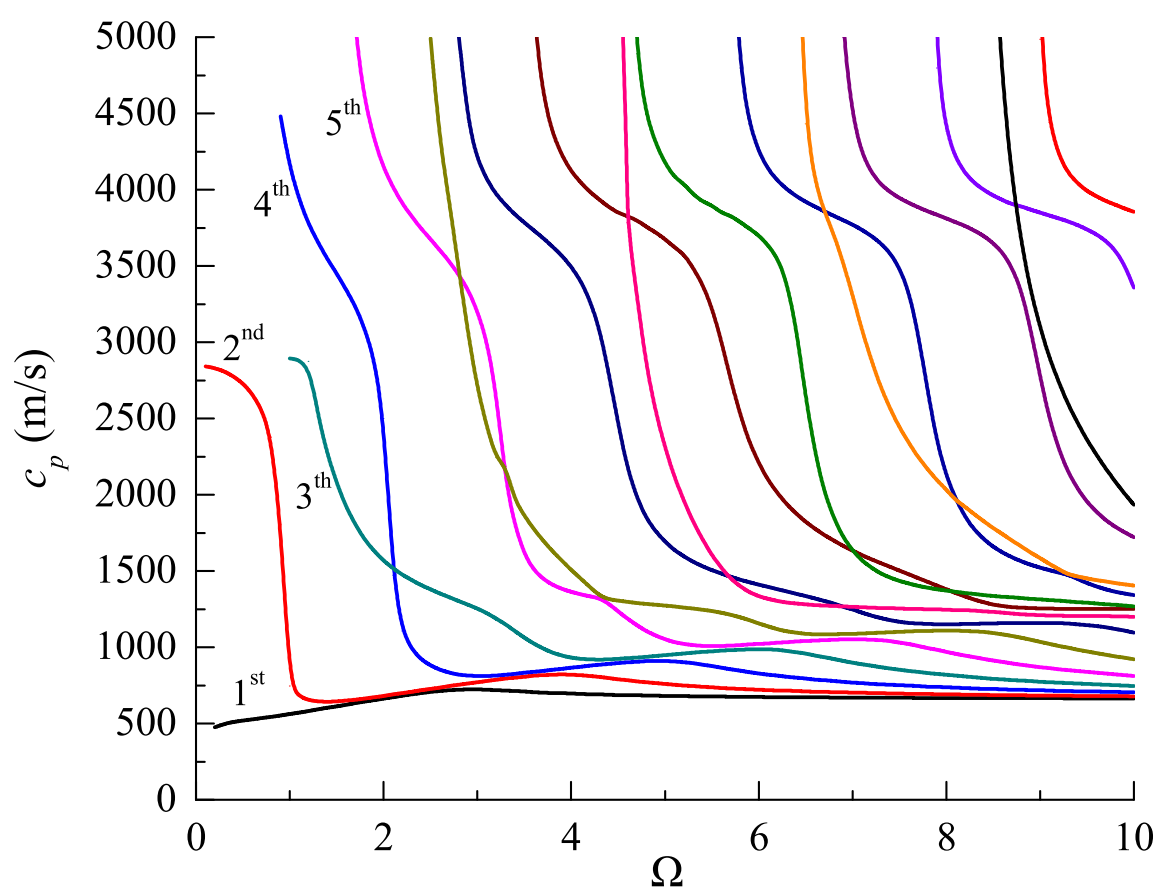

(b) Phase velocity curves 


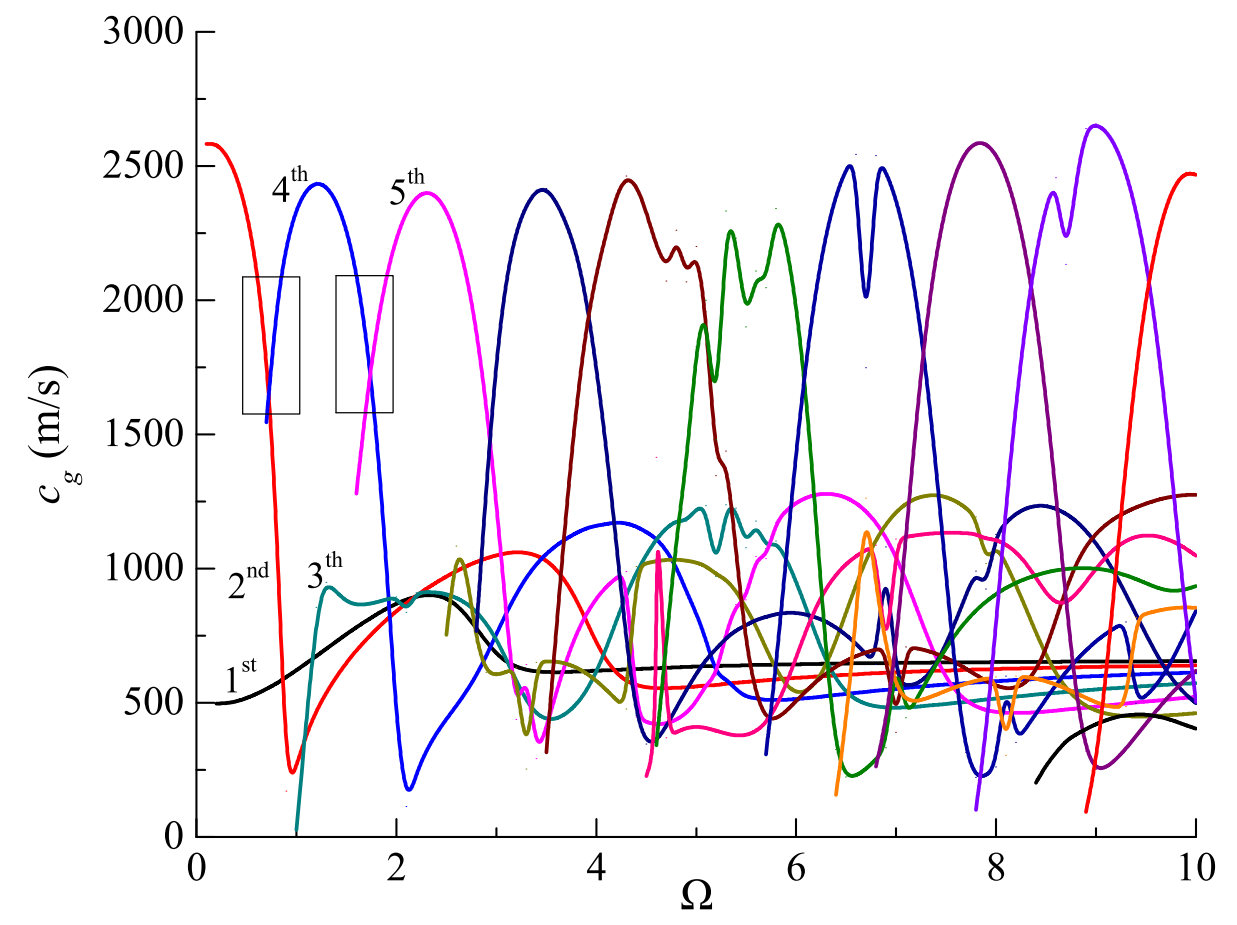

(c) Group velocity curves

Fig. 5. Dispersive curves of $\mathrm{Cu}-\mathrm{PVDF}-\mathrm{Cu}$ waveguides

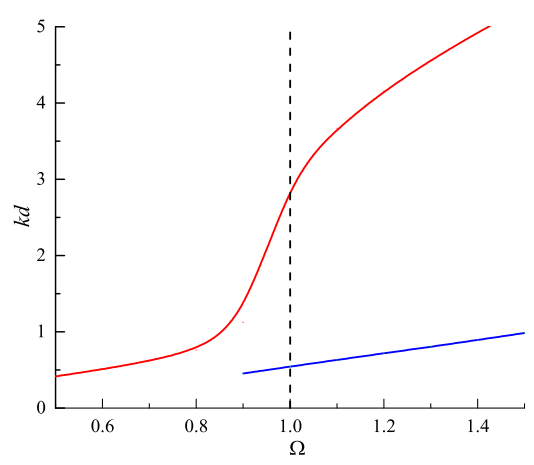

(a) Spectrum curves

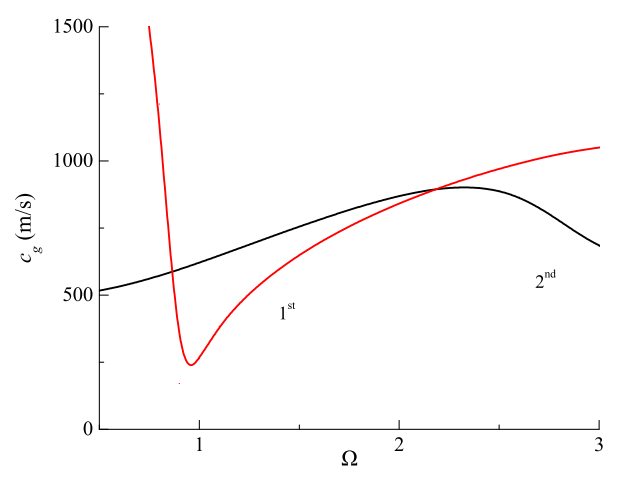

(b) Group velocity curves

Fig. 6. Dispersive curves for $\Omega$ ranging from 05 and 1.5 


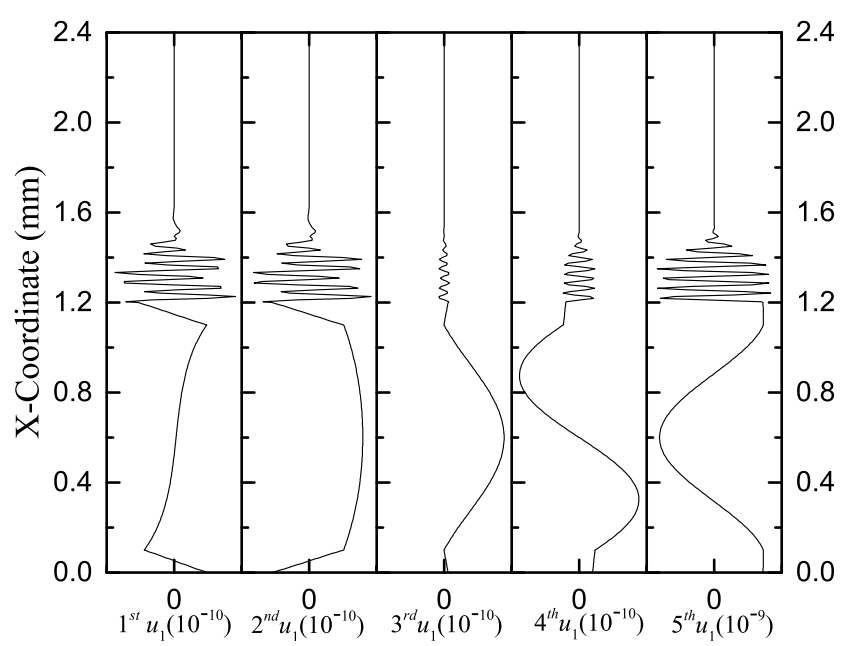

(a) $u_{1}$ displacement

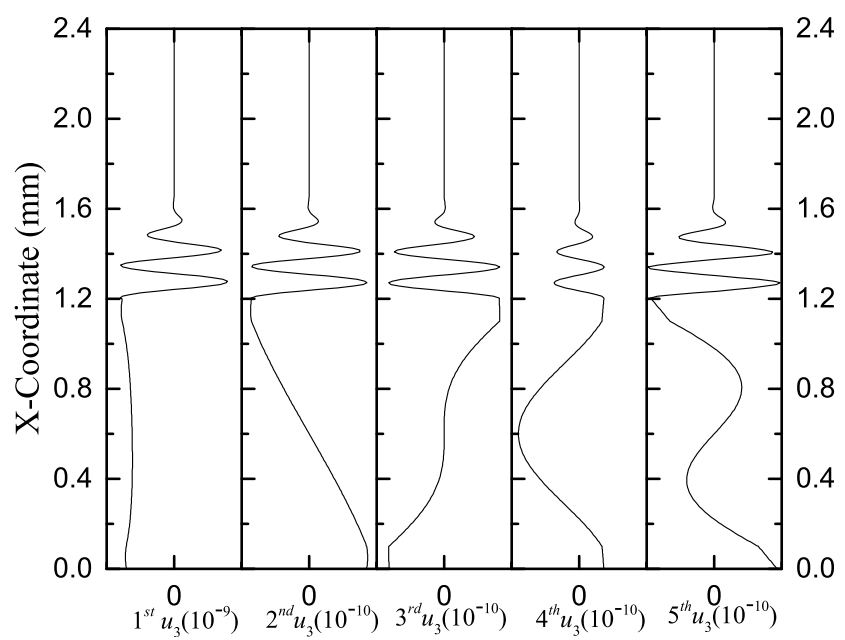

(b) $u_{3}$ displacement

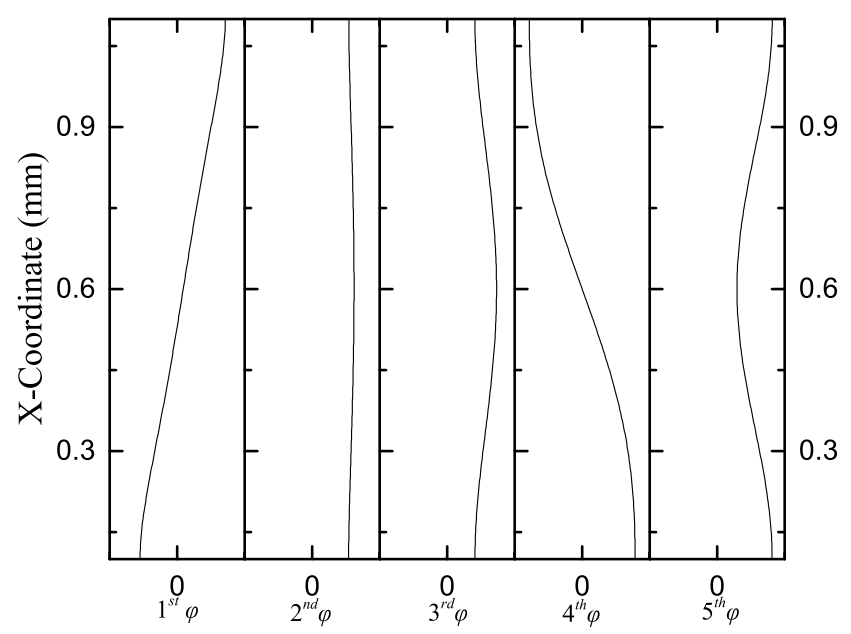

(c) Electric potential $\phi$

Fig. 7. Wave structures of first-five modes at $\Omega=2$ 


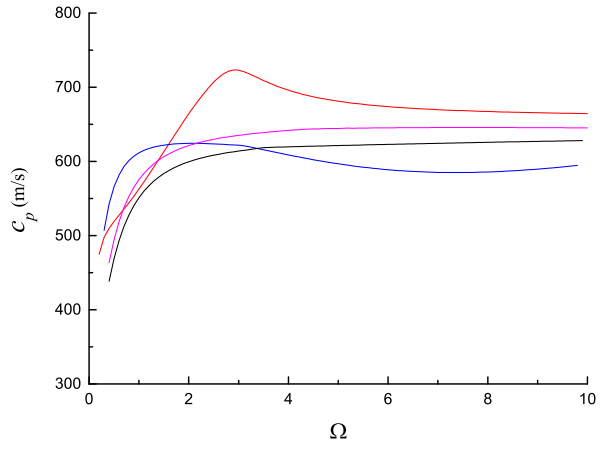

(a) Phase velocity curves of $1^{\text {st }}$ mode

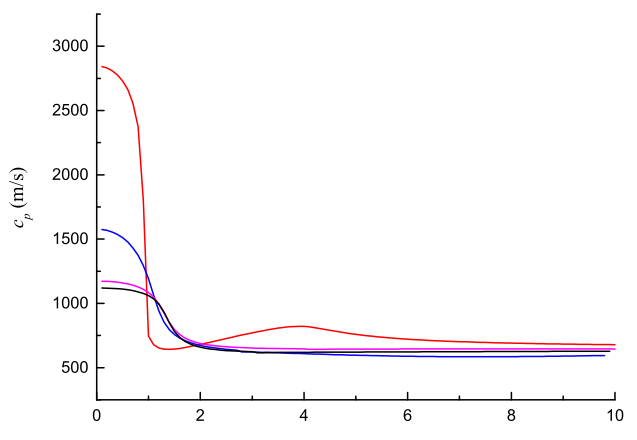

$\Omega$

(c) Phase velocity curves of $2^{\text {nd }}$ mode

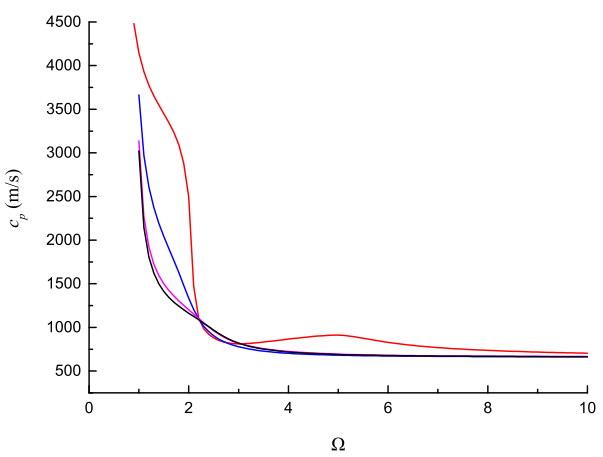

(e) Phase velocity curves of $3^{r d}$ mode

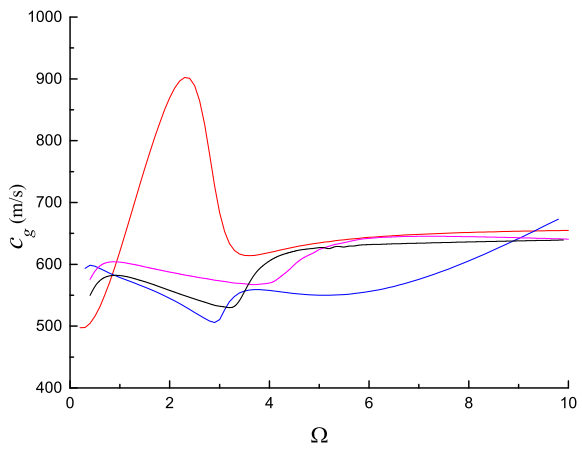

(b) Group velocity curves of $1^{\text {st }}$ mode

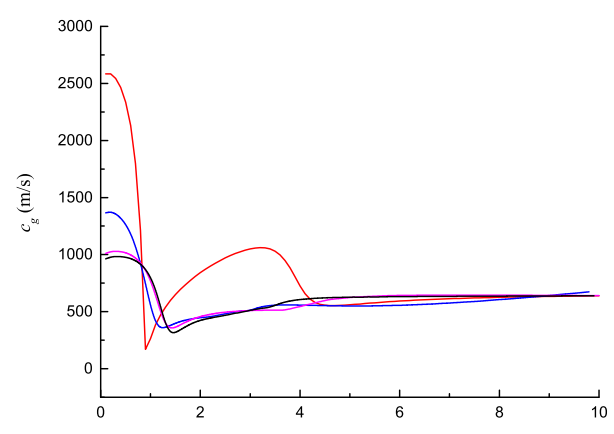

$\Omega$

(d) Group velocity curves of $2^{\text {nd }}$ mode

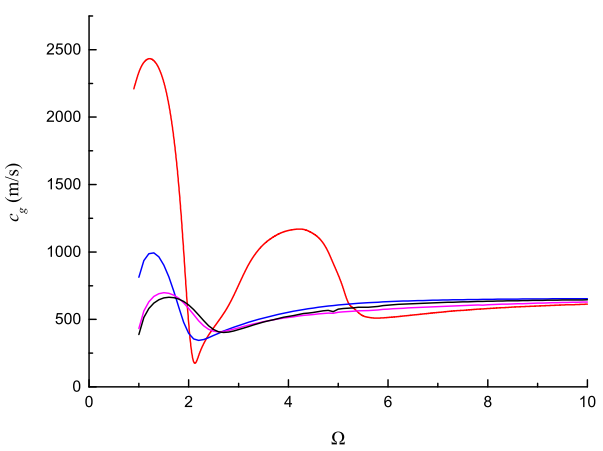

(f) Group velocity curves of $3^{\text {rd }}$ mode

Fig. 8. Dispersive curves of the first-three modes with different Cu's thickness: Red $-0.1 \mathrm{~mm}$, Blue-0.01mm, Magenta$0.01 \mathrm{~mm}$, Black- $0.001 \mathrm{~mm}$ 
Fig.8 presents the phase and group velocity curves vs. frequency curves for difference thickness of $\mathrm{Cu}$ film. Here, the shear velocity of $\mathrm{Cu}$ is more larger than that of others two materials. When the $\mathrm{Cu}$ 's film increases for this waveguide, the structure becomes more stiff. In other words, the phase and group velocities increase with the thickness of $\mathrm{Cu}$ 's film increasing, in a frequency region for $0<\Omega<2$. In addition, it can be clearly observed that phase and group velocity of the first-three modes are fairly flat in a high frequency region. The first-three modes of this frequency region are well suitable to piratical use of SAWs. Addition to, Compared to others color curves, the red curve becomes more different because of modal transition between the $1^{s} t$ and $2^{\text {nd }}$ and mode.

The manufacturing technique has great effect on the elastic modulus of PDMS. Thereby, the impact of PDMSs $E$ on dispersive curves are required to analyze. Dispersive curves of different PDMS elastic modulus are almost identical, without altering any other material parameters and geometry of waveguides. Hence, PDMS's elastic modulus have very small effect on surface wave propagation of $\mathrm{Cu}-\mathrm{PVDF}-\mathrm{Cu}$ waveguide. In the variable range, PDMS's elastic modulus are always rather small than that of PVDF, so that the change of PDMS's elastic modulus can not generate the trend of dispersive curves.

\section{Conclusions}

In this paper, the SIGA-PML formulation related to the piezoelectric layer with a substrate is presented to analyze dispersion properties of Rayleigh waves. The proposed method combines the advantages of IGA and PML. the PML technique is employed to vanish the reflections of wave. The NURBS elements of SIGA have basic functions with higher continuity, compared with traditional elements, so that this method can achieve more accuracy and effective results than SAFE-PML. Finally, we plot dispersive curves and wave structures of $\mathrm{Cu}-\mathrm{PVDF}-\mathrm{Cu}$ waveguides and further discuss propagation properties of Rayleigh waves. And, We discover that the effect of PDMS elastic modulus are small on dispersive behaviors, while the impact of Cu's thickness on wave propagation of the first-three modes turn much more obvious.

\section{Acknowledgements}

The authors wish to acknowledge the support from by the National Natural Science Foundation of China (Grant No. 11702067), Guangdong Province (Grant No.2016A030313617 and and No.2018A030310310), and the Foundation for Young Talents in Higher Education of Guangdong, China (Grant No. 2015KQNCX122)

\section{Appendix A}

The generally displacement, strain and stress fields of RayleigBleustein1968Ah wave, respectively, are given as

$$
\begin{aligned}
\overline{\boldsymbol{u}} & =\left[\begin{array}{llll}
u_{1} & u_{3} & -\phi
\end{array}\right]^{T} \\
\overline{\boldsymbol{\sigma}} & =\left[\begin{array}{lllll}
\sigma_{11} & \sigma_{22} & \sigma_{12} & D_{1} & D_{2}
\end{array}\right]^{T} \\
\overline{\boldsymbol{\varepsilon}} & =\left[\begin{array}{lllll}
\varepsilon_{11} & \varepsilon_{22} & \varepsilon_{12} & E_{1} & E_{2}
\end{array}\right]^{T}
\end{aligned}
$$

Their corresponding differential operator are expressed as

$$
\mathbf{L}_{x}=\left[\begin{array}{ccc}
1 & 0 & 0 \\
0 & 0 & 0 \\
0 & 1 & 0 \\
0 & 0 & -1 \\
0 & 0 & 0
\end{array}\right], \quad \mathbf{L}_{y}=\left[\begin{array}{ccc}
0 & 0 & 0 \\
0 & 1 & 0 \\
1 & 0 & 0 \\
0 & 0 & 0 \\
0 & 0 & -1
\end{array}\right]
$$


Their constitutive parameters are described as

$$
\mathbf{C}=\left[\begin{array}{ccc}
c_{11} & c_{13} & 0 \\
c_{31} & c_{33} & 0 \\
0 & 0 & c_{44}
\end{array}\right], \quad \mathbf{e}=\left[\begin{array}{ccc}
e_{31} & e_{33} & 0 \\
0 & 0 & e_{15}
\end{array}\right], \quad \mathbf{v}=\left[\begin{array}{cc}
v_{11} & 0 \\
0 & v_{33}
\end{array}\right]
$$

\section{References}

[1] R. M. White, F. W. Voltmer, Direct piezoelectric coupling to surface elastic waves, Applied Physics Letters 7 (12) (1965) 314-316.

[2] J. Friend, L. Y. Yeo, Microscale acoustofluidics: Microfluidics driven via acoustics and ultrasonics, Reviews of Modern Physics 83 (2) (2011) 647-704.

[3] F. Guo, Z. Mao, Y. Chen, Z. Xie, J. P. Lata, P. Li, L. Ren, J. Liu, J. Yang, M. Dao, Threedimensional manipulation of single cells using surface acoustic waves, Proceedings of the National Academy of Sciences of the United States of America 113 (6) (2016) 1522.

[4] C. Devendran, D. J. Collins, Y. Ai, A. Neild, Huygens-fresnel acoustic interference and the development of robust time-averaged patterns from traveling surface acoustic waves, Physical Review Letters 118 (15) (2017) 154501.

[5] J. L. Rose, Ultrasonic guided waves in solid media, Cambridge University Press, 2014.

[6] N. E. Glass, R. Loudon, A. A. Maradudin, Propagation of rayleigh surface waves across a largeamplitude grating, Physical Review B 24 (12) (1981) 6843-6861.

[7] Y. B. Fu, S. L. B. Hill, Propagation of steady nonlinear waves in a coated elastic half-space, Wave Motion 34 (1) (2001) 109-129.

[8] J. L. Bleustein, A new surface wave in piezoelectric materials, Applied Physics Letters 13 (12) (1968) 412-413.

[9] H. Fang, J. Yang, Q. Jiang, Surface acoustic waves propagating over a rotating piezoelectric half-space, IEEE Trans Ultrason Ferroelectr Freq Control 48 (4) (2001) 998-1004.

[10] J. F. Chai, T. T. Wu, Propagation of surface waves in a prestressed piezoelectric material, Journal of the Acoustical Society of America 100 (4) (1996) 2112-2122.

[11] J. Du, X. Jin, J. Wang, K. Xian, Love wave propagation in functionally graded piezoelectric material layer, Ultrasonics 46 (1) (2007) 13-22.

[12] Y. Pang, J. X. Liu, Y. S. Wang, X. F. Zhao, Propagation of rayleigh-type surface waves in a transversely isotropic piezoelectric layer on a piezomagnetic half-space, Journal of Applied Physics $103(7)(2008) 770$.

[13] X. Cao, F. Jin, Z. Wang, On dispersion relations of rayleigh waves in a functionally graded piezoelectric material (fgpm) half-space, Acta Mechanica 200 (3-4) (2008) 247-261.

[14] X. Cao, F. Jin, I. Jeon, Rayleigh surface wave in a piezoelectric wafer with subsurface damage, Applied Physics Letters 95 (26) (2009) 239.

[15] O. B. Matar, N. Gasmi, H. Zhou, M. Goueygou, A. Talbi, Legendre and laguerre polynomial approach for modeling of wave propagation in layered magneto-electro-elastic media, Journal of the Acoustical Society of America 133 (3) (2013) 1415-1424.

[16] S. Zhang, B. Gu, H. Zhang, X. Q. Feng, R. Pan, Alamusi, N. Hu, Propagation of love waves with surface effects in an electrically-shorted piezoelectric nanofilm on a half-space elastic substrate, Ultrasonics 66 (2016) 65-71. 
[17] C. Enzevaee, H. M. Shodja, Crystallography and surface effects on the propagation of love and rayleigh surface waves in fcc semi-infinite solids, International Journal of Solids and Structures 138.

[18] Y. Liu, Q. Han, Y. Liang, G. Xu, Numerical investigation of dispersive behaviors for helical thread waveguides using the semi-analytical isogeometric analysis method., Ultrasonics 83.

[19] H. Gravenkamp, S. Natarajan, W. Dornisch, On the use of nurbs-based discretizations in the scaled boundary finite element method for wave propagation problems, Computer Methods in Applied Mechanics and Engineering 315 (2017) 867-880.

[20] T. J. R. Hughes, J. A. Cottrell, Y. Bazilevs, Isogeometric analysis: Cad, finite elements, nurbs, exact geometry and mesh refinement, Computer Methods in Applied Mechanics and Engineering 194 (39) (2005) 4135-4195.

[21] J. A. Cottrell, A. Reali, Y. Bazilevs, T. J. R. Hughes, Isogeometric analysis of structural vibrations, Computer Methods in Applied Mechanics and Engineering 195 (41) (2006) 5257-5296.

[22] J. A. Cottrell, T. J. R. Hughes, Y. Bazilevs, Isogeometric Analysis: Toward Integration of CAD and FEA, Wiley Publishing, 2009.

[23] F. Treyssde, A. Frikha, P. Cartraud, Mechanical modeling of helical structures accounting for translational invariance. part 2 : Guided wave propagation under axial loads, International Journal of Solids and Structures 50 (9) (2013) 1383-1393.

[24] N. Ryden, M. J. S. Lowe, Guided wave propagation in three-layer pavement structures, Journal of the Acoustical Society of America 116 (5) (2004) 2902-2913.

[25] M. Castaings, M. Lowe, Finite element model for waves guided along solid systems of arbitrary section coupled to infinite solid media, Journal of the Acoustical Society of America 123 (2) (2008) 696.

[26] Z. Fan, M. J. S. Lowe, M. Castaings, C. Bacon, Torsional waves propagation along a waveguide of arbitrary cross section immersed in a perfect fluid, Journal of the Acoustical Society of America 124 (4) (2008) 2002-10.

[27] M. Mazzotti, I. Bartoli, A. Marzani, E. Viola, A coupled safe-2.5d bem approach for the dispersion analysis of damped leaky guided waves in embedded waveguides of arbitrary cross-section, Ultrasonics 53 (7) (2013) 1227-1241.

[28] M. Mazzotti, A. Marzani, I. Bartoli, Dispersion analysis of leaky guided waves in fluid-loaded waveguides of generic shape, Ultrasonics 54 (1) (2014) 408-418.

[29] F. Treyssde, K. L. Nguyen, A. S. Bonnet-Bendhia, C. Hazard, Finite element computation of trapped and leaky elastic waves in open stratified waveguides, Wave Motion 51 (7) (2014) 10931107.

[30] K. L. Nguyen, F. Treyssde, C. Hazard, Numerical modeling of three-dimensional open elastic waveguides combining semi-analytical finite element and perfectly matched layer methods, Journal of Sound and Vibration 344 (2015) 158-178.

[31] F. Treyssde, Spectral element computation of high-frequency leaky modes in three-dimensional solid waveguides, Journal of Computational Physics 314 (2016) 341-354. 